\title{
带内取向柔性多醚链苯炔大环合成
}

\author{
李全* 郑盈王力易守兵程晓红* \\ (云南大学化学科学与工程学院 教育部自然资源药物化学重点实验室 昆明 650091)
}

\begin{abstract}
摘要 采用分子间 Glaser 半环闭环法合成了带内取向柔性多醚链的苯炔大环. 用 ${ }^{1} \mathrm{H} N M R,{ }^{13} \mathrm{C}$ NMR, HRMS, UV 及 PL (photduminescence) 确证了目标大环结构, 凝胶色谱测定了目标大环纯度. 经偏光显微镜(POM)和差热分析仪(DSC)测 试表明大环没有呈现预期的液晶性质, 可能是由于环内柔性链过于拥挤, 不能形成与环平面共面结构, 以至于难于进 行有序堆积的缘故.
\end{abstract}

关键词 苯炔大环; 合成; 内取向; 多醚链

\section{Synthesis of Shape-Persistent Phenyl Ethynylene Macrocycles with Intrannular Flexible Oligooxylethylene Chains}

\author{
Li, Quan* Zheng, Ying Wang, Li Yi, Shoubing Cheng, Xiaohong* \\ (School of Chemistry Science and Engineering, Key Laboratory of Medicinal Chemistry for Natural Resourse of \\ Ministry of Education, Yunnan University, Kunming 650091)
}

\begin{abstract}
Shape-persistent phenyl ethynylene macrocycles with intraanular flexible oligooxylethylene chains were synthesized by intramolecular Glaser coupling reaction of half rings. The structures of target phenyl ethynylene macrocycles were identified by ${ }^{1} \mathrm{H}$ NMR, ${ }^{13} \mathrm{C}$ NMR, HRMS, UV and PL (photduminescence), The purity of the target macrocycles was determined by GPC. All the macrocycles are nomesogens as indicated by polarized optical microscopy (POM) and differential scanning calorimetry (DSC), the reason is analyzed as the streric hindrance of the intrannular flexible chains which led to the anordered aggregation of the macrocycles.
\end{abstract}

Keywords phenyl ethynylene macrocycle; synthesis; intrannular; oligooxylethylene chain

苯炔大环分子环骨架由苯基和乙炔基单元构成, 具 有规整的几何构型，环内直径可达到纳米尺寸，环平面 上有大 $\pi$ 电子共轭体系, 苯环上可带各种官能团, 其分 子平面间可通过 $\pi-\pi$ 键作用、溶剂化作用、苯环上极性 官能团的相互作用等 ${ }^{[1]}$ 自组装成特定的超分子结构 ${ }^{[24]}$, 形成热力学液晶 ${ }^{[5,6]}$, 其纳米尺寸直径的环内空腔可应 用于主客体分子识别 ${ }^{[7,8]}$, 因此其合成是近年研究热 点 ${ }^{[9 \sim 14]}$, 自 Staab 等 ${ }^{[15]}$ 用苯炔单体一步合成苯炔大环后, 苯炔大环的合成发展了逐步预聚 $\alpha, \omega$-齐聚物闭环法、模 板合成法、半环对接闭环法、相似结构苯烯环脱氢法等. Höger 小组 ${ }^{[16]}$ 合成了带内取向烷氧基链的苯炔大环, 发 现具有向列相液晶性质, 初探了这种具有反拓朴结构的 分子产生和不产生向列相液晶的原因 ${ }^{[17,18]}$, 提出了该类 分子获得液晶的设计预备性原则, 但分子结构与液晶性 质的关系仍不明朗. 本文依据 Höger 等提出的预备性原
则, 采用半环对接闭环法设计合成了带六条内取向柔性 链的苯炔大环，引入了寡聚乙氧基极性多醚链，希望利 用多醚链间产生的额外相互作用来增加分子间的堆积 作用, 获取液晶相结构. 遗憾的是本文所合成的大环经 偏光显微镜(POM)和差热分析仪(DSC)测试并没有呈现 所期待的液晶性质. 本文根据这类反拓扑结构的分子非 簇集倾向性的特点，分析了它们不能形成液晶的原因， 为今后相关的研究提供了理论指导和实验数据. 所合成 的结构未见文献报道, 目标大环结构通过了 ${ }^{1} \mathrm{H}$ NMR, ${ }^{13} \mathrm{C}$ NMR, HRMS, UV 和 PL (photduminescence) 证实, 并 且用凝胶色谱对目标大环纯度进行了测定.

\section{1 实验部分}

\section{1 仪器与试剂}

AV.drx500 型核磁共振仪(TMS 为内标); Agilent

*E-mail: leequan@ynu.edu.cn; xhcheng@ynu.edu.cn

Received January 10, 2011; revised Sepember 14, 2011; accepted Sepember 27, 2011.

Project supported by the Ph.D. Program Foundation of Ministry of Education of China (No. 20070673002).

国家教育部博士点基金(No. 20070673002)资助项目. 


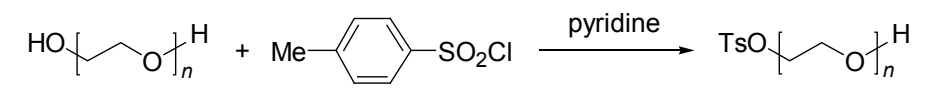

1a: $n=2,1 b: n=3$
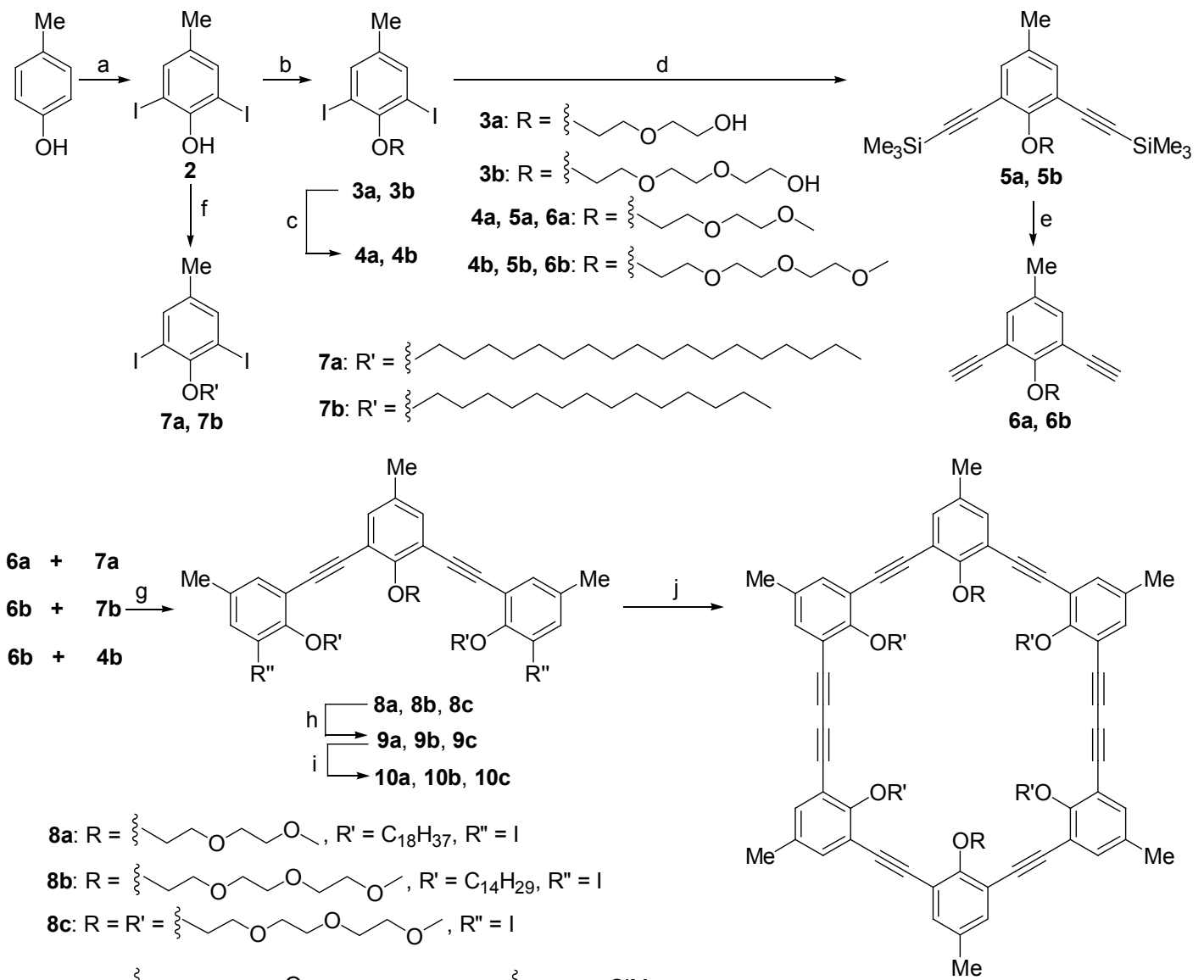

9a: $\mathrm{R}=\xi \smile \mathrm{O} \sim \mathrm{R}^{\prime}=\mathrm{C}_{18} \mathrm{H}_{37}, \mathrm{R}^{\prime \prime}=\xi \overline{\mathrm{O}} \mathrm{SiMe}_{3}$

9b: $\mathrm{R}=\xi \mathrm{O}_{\mathrm{O}} \mathrm{O}^{\prime}=\mathrm{C}_{14} \mathrm{H}_{29}, \mathrm{R}^{\prime \prime}=\xi \overline{\mathrm{O}}=\mathrm{SiMe}_{3}$

11a, 11b, 11c

9c: $\left.\mathrm{R}=\mathrm{R}^{\prime}=\xi\right) \mathrm{SiMe}_{3}$

11a: $\mathrm{R}=\mathrm{\zeta}_{\mathrm{O}} \bigcirc, \mathrm{R}^{\prime}=\mathrm{C}_{18} \mathrm{H}_{37}$

10a: $\mathrm{R}=\xi \mathrm{O}_{\backslash}, \mathrm{R}^{\prime}=\mathrm{C}_{18} \mathrm{H}_{37}, \mathrm{R}^{\prime}=\xi \overline{\overline{ }}$

10b: $\mathrm{R}=\xi \backslash \mathrm{O}_{\mathrm{O}} \mathrm{R}^{\prime}=\mathrm{C}_{14} \mathrm{H}_{29}, \mathrm{R}^{\prime \prime}=\xi$

11b: $\mathrm{R}=\xi \mathrm{O}^{\prime}, \mathrm{R}^{\prime}=\mathrm{C}_{14} \mathrm{H}_{29}$

10c: $\mathrm{R}=\mathrm{R}^{\prime}=\xi$

11c: $\mathrm{R}=\mathrm{R}^{\prime}=\xi^{\prime}$

Reagents and conditions: (a) $\mathrm{MeOH}, \mathrm{NH}_{4} \mathrm{OH}, \mathrm{I}_{2}, \mathrm{KI}, \mathrm{H}_{2} \mathrm{O}$; (b) 1a or 1b, MeCN, $\mathrm{K}_{2} \mathrm{CO}_{3}$; (c) NaH, DMF, Mel; (d) $\mathrm{Pd}_{(}\left(\mathrm{PPh}_{3}\right)_{2} \mathrm{Cl}_{2}, \mathrm{Cul}, \mathrm{PPh}_{3}, \mathrm{THF}$, triethylamine, $\equiv \mathrm{SiMe}_{3}$; (e) $\mathrm{K}_{2} \mathrm{CO}_{3}, \mathrm{MeOH}$, THF; (f) R'Br, DMF, $\mathrm{K}_{2} \mathrm{CO}_{3}$; (g) $\mathrm{Pd}\left(\mathrm{PPh}_{3}\right)_{2} \mathrm{Cl}_{2}, \mathrm{Cul}_{1} \mathrm{PPh}_{3}$, THF, triethylamine; (h) $\mathrm{Pd}(\mathrm{PPh})_{2} \mathrm{Cl}_{2}$, Cul, $\mathrm{PPh}_{3}$, THF, triethylamine, $\equiv \mathrm{SiMe}_{3}$; (i) $\mathrm{K}_{2} \mathrm{CO}_{3}, \mathrm{MeOH}$, THF; (j) $\mathrm{CuCl}, \mathrm{CuCl}_{2}$, pyridine

\section{Scheme 1}

LC/MSD TOF 6210 质谱仪(ESI 离子源); Shimadzu UV-2401PC 紫外可见分光光度计(日本岛津); Hitch F-4500FL 型苂光光谱仪(日本日立); Perkin-Elmer DSC-7 差热分析仪; CF-XMT-3100 偏光显微镜(上海长方光学 仪器厂); Agilent 1100 凝胶渗透色谱仪(THF 流动相, 1.0 $\mathrm{mL} / \mathrm{min}, 25{ }^{\circ} \mathrm{C}$ ); 试剂均为市售分析纯或化学纯试剂.

\section{2 二乙二醇对甲苯磺酸单酯(1a)和三乙二醇对甲苯} 磺酸单酯 $(1 b)$ 的合成

在 $100 \mathrm{~mL}$ 单口烧瓶中加入 $100 \mathrm{mmol}$ 三乙二醇或二 乙二醇、 $25 \mathrm{~mL}$ 吡啶, 冰水冷却并搅拌下加入对甲苯磺
酰氯 $20 \mathrm{mmol}$, 室温摚拌 $5 \mathrm{~h}$, 加入适量乙酯乙酯, $1: 1$ 盐酸洗涤至呈酸性，乙酸乙酯层干燥、蒸干即得 $1 \mathbf{a}$ 或 $1 \mathbf{b}$ (下一步反应直接使用).

\subsection{4-甲基-2,6-二碘苯酚(2)的合成}

在 $500 \mathrm{~mL}$ 单口烧瓶中加入 $9.914 \mathrm{~g}(91.68 \mathrm{mmol})$ 对 甲苯酚、 $79 \mathrm{~mL}$ 氨水、 $51 \mathrm{~mL}$ 甲醇, 慢慢滴入含碘 $50.8 \mathrm{~g}$ (200.00 mmol), KI $78.874 \mathrm{~g}$ ，水 $130 \mathrm{~mL}$ 溶液(滴入的碘颜 色不再消失时，应停止滴入)，过滤，固体用 $V$ (石油 醚) $: V($ 乙酸乙酯 $)=10 ： 1$ 作洗脱剂柱层析得白色针状 晶体 $13.062 \mathrm{~g}$ ，以对甲苯酚计收率 $39.6 \%$. ${ }^{1} \mathrm{H}$ NMR 
$\left(\mathrm{CDCl}_{3}, 500 \mathrm{~Hz}\right) \delta: 2.22\left(\mathrm{~s}, 3 \mathrm{H}, \mathrm{Ar}-\mathrm{CH}_{3}\right), 2.47$ (brs, $1 \mathrm{H}$, $\mathrm{OH}), 7.49$ (s, 2H, $2 \times \mathrm{Ar}-\mathrm{H})$.

1.4 二乙二醇单-(4-甲基-2,6-二碘苯酚基)醚(3a)和三 乙二醇单-(4-甲基-2,6-二磑苯酚基)醚(3b)的合成

在 $100 \mathrm{~mL}$ 单口烧瓶中加入 $23.602 \mathrm{~g}(10.01 \mathrm{mmol})$, 1a $3.902 \mathrm{~g}$ (15.01 mmol, 第 1.2 节合成的产物直接使用), 乙腈 $35 \mathrm{~mL}$, 无水 $\mathrm{K}_{2} \mathrm{CO}_{3} 2.123 \mathrm{~g}(15.38 \mathrm{mmol})$, 四丁基 溴化铵少许, 加热搅拌回流 $5 \mathrm{~h}$, 过滤, 滤液蒸干, 柱层 析得到 3a $2.879 \mathrm{~g}$, 以 2 计收率 64.20\%. ${ }^{1} \mathrm{H} \mathrm{NMR}\left(\mathrm{CDCl}_{3}\right.$, $500 \mathrm{~Hz}) \delta: 2.20$ (s, 3H, Ar- $\left.\mathrm{CH}_{3}\right), 2.70$ (brs, $\left.1 \mathrm{H}, \mathrm{OH}\right), 3.71$ (t, $J=4.5 \mathrm{~Hz}, 2 \mathrm{H}, \mathrm{CH}_{2}$ ), 3.76 (t, $J=4.1 \mathrm{~Hz}, 2 \mathrm{H}, \mathrm{CH}_{2}$ ), 3.95 (t, $\left.J=4.9 \mathrm{~Hz}, 2 \mathrm{H}, \mathrm{CH}_{2}\right), 4.09\left(\mathrm{t}, J=4.7 \mathrm{~Hz}, 2 \mathrm{H}, \mathrm{CH}_{2}\right), 7.54$ (s, $2 \mathrm{H}, 2 \times \mathrm{Ar}-\mathrm{H}) ;{ }^{13} \mathrm{C} \mathrm{NMR}\left(\mathrm{CDCl}_{3}, 125 \mathrm{~Hz}\right) \delta: 20.10(1 \mathrm{C}$, Ar- $\left.\mathrm{CH}_{3}\right), 62.23,70.51,72.57,73.00\left(4 \mathrm{C}, \mathrm{OCH}_{2}\right), 90.83$, $138.30,140.74,155.73$ (6C, 苯环).

参照上述操作, $23.598 \mathrm{~g}$ (10.0 mmol), $1 \mathrm{~b} 4.565 \mathrm{~g}$ (15.02 mmol) 得 3b $3.833 \mathrm{~g}$, 以 2 计收率 77.91\%. ${ }^{1} \mathrm{H}$ NMR $\left(\mathrm{CDCl}_{3}, 500 \mathrm{~Hz}\right) \delta: 2.23$ (s, 3H, Ar- $\left.\mathrm{CH}_{3}\right), 2.69$ (brs, $1 \mathrm{H}$, $\mathrm{OH}), 3.64\left(\mathrm{t}, J=4.7 \mathrm{~Hz}, 2 \mathrm{H}, \mathrm{CH}_{2}\right), 3.74\left(\mathrm{br}, 4 \mathrm{H}, 2 \times \mathrm{CH}_{2}\right.$ ), $3.82\left(\mathrm{t}, J=4.1 \mathrm{~Hz}, 2 \mathrm{H}, \mathrm{CH}_{2}\right), 3.98\left(\mathrm{t}, J=5.0 \mathrm{~Hz}, 2 \mathrm{H}, \mathrm{CH}_{2}\right.$ ), $4.13\left(\mathrm{t}, J=4.9 \mathrm{~Hz}, 2 \mathrm{H}, \mathrm{CH}_{2}\right), 7.58(\mathrm{~s}, 2 \mathrm{H}, 2 \times \mathrm{Ar}-\mathrm{H}) ;{ }^{13} \mathrm{C}$ NMR $\left(\mathrm{CDCl}_{3}, 125 \mathrm{~Hz}\right) \delta: 20.04\left(1 \mathrm{C}, \mathrm{Ar}-\mathrm{CH}_{3}\right), 62.20$, $70.58,70.91,71.31,72.56,72.98\left(6 \mathrm{C}, \mathrm{OCH}_{2}\right), 90.76$, $138.24,140.74,155.86$ (6C, 苯环).

\section{5 二乙二醇-(4-甲基-2,6-二磑苯酚基)甲醚(4a)和三} 乙二醇-(4-甲基-2,6-二碘苯酚基)甲醚(4b)的合成

在 $50 \mathrm{~mL}$ 加入 $3 \mathrm{a} 2.158 \mathrm{~g}(4.82 \mathrm{mmol})$, 无水 DMF 13 $\mathrm{mL}$, 冰水冷却下, 加入氢化钠 $(60 \%) 0.311 \mathrm{~g}(7.78 \mathrm{mmol})$, 搅拌 $5 \mathrm{~min}$, 加入 $\mathrm{CH}_{3} \mathrm{I} 1.023 \mathrm{~g}(7.20 \mathrm{mmol})$, 室温摚拌 5 $\mathrm{h}$, 加入适量水, 乙酸乙酯萃取, 柱层析得无色液体 $\mathbf{4 a}$ $2.130 \mathrm{~g}$, 收率 $95.65 \% .{ }^{1} \mathrm{H}$ NMR $\left(\mathrm{CDCl}_{3}, 500 \mathrm{~Hz}\right) \delta: 2.21$ (s, $\left.3 \mathrm{H}, \mathrm{Ar}_{-} \mathrm{CH}_{3}\right), 3.40$ (s, $3 \mathrm{H}, \mathrm{OCH}_{3}$ ), 3.60 (t, $J=4.8 \mathrm{~Hz}$, $2 \mathrm{H}, \mathrm{CH}_{2}$ ), 3.79 (t, $J=5.0 \mathrm{~Hz}, 2 \mathrm{H}, \mathrm{CH}_{2}$ ), 3.97 (t, $J=5.2 \mathrm{~Hz}$, $\left.2 \mathrm{H}, \mathrm{CH}_{2}\right), 4.12\left(\mathrm{t}, J=5.0 \mathrm{~Hz}, 2 \mathrm{H}, \mathrm{CH}_{2}\right), 7.56(\mathrm{~s}, 2 \mathrm{H}, 2 \times$ Ar-H).

参照上述操作, 3b $2.327 \mathrm{~g}(4.73 \mathrm{mmol})$ 得无色液体 4b $2.392 \mathrm{~g}$, 收率 $100.00 \% .{ }^{1} \mathrm{H}$ NMR $\left(\mathrm{CDCl}_{3}, 500 \mathrm{~Hz}\right) \delta$ : 2.24 (s, $\left.3 \mathrm{H}, \mathrm{Ar}-\mathrm{CH}_{3}\right), 3.39$ (s, $\left.3 \mathrm{H}, \mathrm{OCH}_{3}\right), 3.57$ (t, $J=5.0$ $\left.\mathrm{Hz}, 2 \mathrm{H}, \mathrm{CH}_{2}\right), 3.65 \sim 3.79\left(\mathrm{br}, 4 \mathrm{H}, 2 \times \mathrm{CH}_{2}\right), 3.82(\mathrm{t}, J=$ $\left.4.8 \mathrm{~Hz}, 2 \mathrm{H}, \mathrm{CH}_{2}\right), 3.98\left(\mathrm{t}, J=5.0 \mathrm{~Hz}, 2 \mathrm{H}, \mathrm{CH}_{2}\right), 4.13$ (t, $J=$ $\left.5.0 \mathrm{~Hz}, 2 \mathrm{H}, \mathrm{CH}_{2}\right), 7.58(\mathrm{~s}, 2 \mathrm{H}, 2 \times \mathrm{Ar}-\mathrm{H}) ;{ }^{13} \mathrm{C} \mathrm{NMR}\left(\mathrm{CDCl}_{3}\right.$, $125 \mathrm{~Hz}) \delta: 20.03\left(1 \mathrm{C}, \mathrm{Ar}-\mathrm{CH}_{3}\right), 59.46\left(1 \mathrm{C}, \mathrm{OCH}_{3}\right), 70.51$, $71.04,71.14,71.30,72.40,72.55\left(6 \mathrm{C}, \mathrm{OCH}_{2}\right), 90.77$, $138.15,140.72,155.93$ (6C, 苯环).
1.6 二乙二醇-[4-甲基-2,6-二(2-三甲基硅基-1-乙炔基) 苯酚基]甲醚(5a)和三乙二醇-[4-甲基-2,6-二(2-三甲基硅 基-1-乙炔基)苯酚基]甲醚(5b)的合成

在 $50 \mathrm{~mL}$ 单口烧瓶中加入 $4 \mathrm{a} 1.010 \mathrm{~g}(2.19 \mathrm{mmol})$, 三甲基硅乙炔 $0.658 \mathrm{~g}(6.70 \mathrm{mmol})$, 无水 THF $8 \mathrm{~mL}$, 无 水三乙胺 $6 \mathrm{~mL}$, 通氮气驱赶氧气 $5 \mathrm{~min}$ 后, 加入三苯基 膦 $0.040 \mathrm{~g}$, 二三苯基膦二氯化钯 $0.026 \mathrm{~g}$, 碘化亚铜 $0.018 \mathrm{~g}$, 密闭搅拌 $12 \mathrm{~h}$, 过滤除去沉淀后, 蒸干, 柱层析 得 5a $0.870 \mathrm{~g}$, 收率 $98.82 \% .{ }^{1} \mathrm{H}$ NMR $\left(\mathrm{CDCl}_{3}, 500 \mathrm{~Hz}\right) \delta$ : $0.24\left(\mathrm{~s}, 12 \mathrm{H}, 3 \times \mathrm{Si}^{-\mathrm{CH}_{3}}\right), 2.21$ (s, $\left.3 \mathrm{H}, \mathrm{Ar}^{-\mathrm{CH}_{3}}\right), 3.37$ (s, $\left.3 \mathrm{H}, \mathrm{OCH}_{3}\right), 3.55\left(\mathrm{t}, J=4.7 \mathrm{~Hz}, 2 \mathrm{H}, \mathrm{CH}_{2}\right), 3.73$ (t, $J=5.0$ $\left.\mathrm{Hz}, 2 \mathrm{H}, \mathrm{CH}_{2}\right), 3.88\left(\mathrm{t}, J=5.4 \mathrm{~Hz}, 2 \mathrm{H}, \mathrm{CH}_{2}\right), 4.33$ (t, $J=5.3$ $\left.\mathrm{Hz}, 2 \mathrm{H}, \mathrm{CH}_{2}\right), 7.19$ (s, 2H, $\left.2 \times \mathrm{Ar}-\mathrm{H}\right) ;{ }^{13} \mathrm{C}$ NMR $\left(\mathrm{CDCl}_{3}\right.$, $125 \mathrm{~Hz}) \delta$ : $0.30\left(6 \mathrm{C}, \mathrm{SiCH}_{3}\right), 20.61\left(1 \mathrm{C}, \mathrm{Ar}-\mathrm{CH}_{3}\right), 59.41$ $\left(1 \mathrm{C}, \mathrm{OCH}_{3}\right), 71.04,71.09,72.40,73.35\left(4 \mathrm{C}, \mathrm{OCH}_{2}\right), 99.15$, 101.33 (2C, C(C), 117.51, 133.17134.93, 1600.25 (6C, 苯 环).

参照上述操作, 4b $7.145 \mathrm{~g}(14.13 \mathrm{mmol})$, 三甲基硅 乙炔 $3.618 \mathrm{~g}\left(36.84 \mathrm{mmol}\right.$ ) 得 $5 \mathbf{b} 6.118 \mathrm{~g}$, 收率 $97.08 \% .{ }^{1} \mathrm{H}$ NMR $\left(\mathrm{CDCl}_{3}, 500 \mathrm{~Hz}\right) \delta: 0.25\left(\mathrm{~s}, 12 \mathrm{H}, 3 \times \mathrm{Si}^{\left.-\mathrm{CH}_{3}\right)}, 2.23\right.$ (s, $\left.3 \mathrm{H}, \mathrm{Ar}-\mathrm{CH}_{3}\right), 3.38$ (s, $\left.3 \mathrm{H}, \mathrm{OCH}_{3}\right), 3.55$ (t, $J=5.0 \mathrm{~Hz}$, $\left.2 \mathrm{H}, \mathrm{CH}_{2}\right), 3.65\left(\mathrm{t}, J=4.9 \mathrm{~Hz}, 2 \mathrm{H}, \mathrm{CH}_{2}\right), 3.67(\mathrm{t}, J=5.0 \mathrm{~Hz}$, $\left.2 \mathrm{H}, \mathrm{CH}_{2}\right), 3.75\left(\mathrm{t}, J=4.8 \mathrm{~Hz}, 2 \mathrm{H}, \mathrm{CH}_{2}\right), 3.88(\mathrm{t}, J=5.0 \mathrm{~Hz}$, $\left.2 \mathrm{H}, \mathrm{CH}_{2}\right), 4.34\left(\mathrm{t}, J=5.0 \mathrm{~Hz}, 2 \mathrm{H}, \mathrm{CH}_{2}\right), 7.19(\mathrm{~s}, 2 \mathrm{H}, 2 \times$ Ar-H); ${ }^{13} \mathrm{C}$ NMR $\left(\mathrm{CDCl}_{3}, 125 \mathrm{~Hz}\right) \delta: 0.32\left(6 \mathrm{C}, \mathrm{SiCH}_{3}\right)$, $20.62\left(1 \mathrm{C}, \mathrm{Ar}-\mathrm{CH}_{3}\right), 59.39\left(1 \mathrm{C}, \mathrm{OCH}_{3}\right), 70.95,71.07$, $71.14,71.53,72.34,73.28\left(6 \mathrm{C}, \mathrm{OCH}_{2}\right), 99.14,101.32(2 \mathrm{C}$, $\mathrm{C}(\mathrm{C}), 117.49,133.15,134.90,160.27$ (6C, 苯环).

1.7 二乙二醇-(4-甲基-2,6-二乙炔基苯酚基)甲醚(6a) 和三乙二醇-(4-甲基-2,6-二乙炔基苯酚基)甲醚(6b)的合 成

在 $25 \mathrm{~mL}$ 单口烧瓶中加入 $5 \mathrm{a} 0.870 \mathrm{~g}(2.16 \mathrm{mmol})$, 无水 THF $8 \mathrm{~mL}$, 无水甲醇 $8 \mathrm{~mL}, 2.501 \mathrm{~g}$ 无水碳酸钾, 密 闭搅拌 $1 \mathrm{~h}$, 过滤, 蒸干, 柱层析得淡红色液体 6a 0.554 $\mathrm{g}$, 收率 $99.22 \% .{ }^{1} \mathrm{H} \mathrm{NMR}\left(\mathrm{CDCl}_{3}, 500 \mathrm{~Hz}\right) \delta: 2.25(\mathrm{~s}, 3 \mathrm{H}$, $\left.\mathrm{Ar}-\mathrm{CH}_{3}\right), 3.25(\mathrm{~s}, 2 \mathrm{H}, 2 \times \mathrm{CH}), 3.38\left(\mathrm{~s}, 3 \mathrm{H}, \mathrm{OCH}_{3}\right), 3.56$ (t, $\left.J=4.6 \mathrm{~Hz}, 2 \mathrm{H}, \mathrm{CH}_{2}\right), 3.74\left(\mathrm{t}, J=5.0 \mathrm{~Hz}, 2 \mathrm{H}, \mathrm{CH}_{2}\right), 3.87(\mathrm{t}$, $\left.J=5.1 \mathrm{~Hz}, 2 \mathrm{H}, \mathrm{CH}_{2}\right), 4.36\left(\mathrm{t}, J=5.0 \mathrm{~Hz}, 2 \mathrm{H}, \mathrm{CH}_{2}\right), 7.24(\mathrm{~s}$, $2 \mathrm{H}, 2 \times \mathrm{Ar}-\mathrm{H}) ;{ }^{13} \mathrm{C}$ NMR $\left(\mathrm{CDCl}_{3}, 125 \mathrm{~Hz}\right) \delta: 20.63(1 \mathrm{C}$, $\left.\mathrm{Ar}-\mathrm{CH}_{3}\right), 59.40\left(1 \mathrm{C}, \mathrm{OCH}_{3}\right), 70.83,71.01,72.40,73.66$ $\left(4 \mathrm{C}, \mathrm{OCH}_{2}\right), 79.93(1 \mathrm{C}, \mathrm{CH} \equiv), 82.06(1 \mathrm{C}, \mathrm{C} \equiv), 116.71$, $133.43,135.50,160.55$ (6C, 苯环).

参照上述操作, 5b $0.492 \mathrm{~g}(1.10 \mathrm{mmol}), 1 \mathrm{~g}$ 无水碳 酸钾得淡红色液体 6b $0.319 \mathrm{~g}$, 收率 $96.03 \% .{ }^{1} \mathrm{H}$ NMR $\left(\mathrm{CDCl}_{3}, 500 \mathrm{~Hz}\right) \delta: 2.25$ (s, 3H, Ar-CH $\left.\mathrm{CH}_{3}\right), 3.29$ (s, $2 \mathrm{H}, 2 \times$ 
$\mathrm{CH}), 3.37\left(\mathrm{~s}, 3 \mathrm{H}, \mathrm{OCH}_{3}\right), 3.54\left(\mathrm{t}, J=4.8 \mathrm{~Hz}, 2 \mathrm{H}, \mathrm{CH}_{2}\right)$, $3.60 \sim 3.71\left(\mathrm{br}, 4 \mathrm{H}, 2 \times \mathrm{CH}_{2}\right), 3.75(\mathrm{t}, J=5.0 \mathrm{~Hz}, 2 \mathrm{H}$, $\left.\mathrm{CH}_{2}\right), 3.86\left(\mathrm{t}, J=5.0 \mathrm{~Hz}, 2 \mathrm{H}, \mathrm{CH}_{2}\right), 4.35(\mathrm{t}, J=5.0 \mathrm{~Hz}, 2 \mathrm{H}$, $\left.\mathrm{CH}_{2}\right), 7.24$ (s, 2H, $\left.2 \times \mathrm{Ar}-\mathrm{H}\right) ;{ }^{13} \mathrm{C} \mathrm{NMR}\left(\mathrm{CDCl}_{3}, 125 \mathrm{~Hz}\right) \delta$ : $20.67\left(1 \mathrm{C}, \mathrm{Ar}-\mathrm{CH}_{3}\right), 59.36\left(1 \mathrm{C}, \mathrm{OCH}_{3}\right), 70.81,70.92$, 71.07, 71.12, 72.33, $73.64\left(6 \mathrm{C}, \mathrm{OCH}_{2}\right), 79.89(1 \mathrm{C}, \mathrm{CH} \equiv)$, $82.19(1 \mathrm{C}, \mathrm{C} \equiv), 116.74,133.45,135.47,160.53$ (6C, 苯 环).

\subsection{4-十八烷氧基-2,6-二碘甲苯(7a)和 4-十四烷氧基- $2,6-$ 二碘甲苯 $(7 b)$ 的合成}

在 $50 \mathrm{~mL}$ 单口烧瓶中加入 $27.188 \mathrm{~g}(19.98 \mathrm{mmol})$, DMF $25 \mathrm{~mL}$, 无水碳酸钾 $3.548 \mathrm{~g}(25.71 \mathrm{mmol})$, 搅拌 5 $\min$, 加入溴代十八烷 $6.65 \mathrm{~g}(19.97 \mathrm{mmol})$, 搅拌 $5 \mathrm{~h}$, 转 入分液漏斗, 加入 $50 \mathrm{~mL}$ 水, $50 \mathrm{~mL} \times 3$ 石油醚萃取, 干 燥, 蒸干, 少量硅胶柱层析得无色液体 7a $12.22 \mathrm{~g}$, 收率 $100 \% .{ }^{1} \mathrm{H}$ NMR $\left(\mathrm{CDCl}_{3}, 500 \mathrm{~Hz}\right) \delta: 0.88(\mathrm{t}, J=7.0 \mathrm{~Hz}, 3 \mathrm{H}$, $\left.\mathrm{CH}_{3}\right), 1.18 \sim 1.47$ (br, $\left.28 \mathrm{H}, 14 \times \mathrm{CH}_{2}\right), 1.50 \sim 1.60(\mathrm{~m}, 2 \mathrm{H}$, $\left.\mathrm{CH}_{2}\right), 1.85 \sim 1.95\left(\mathrm{~m}, 2 \mathrm{H}, \mathrm{CH}_{2}\right), 2.23\left(\mathrm{~s}, 3 \mathrm{H}, \mathrm{Ar}-\mathrm{CH}_{3}\right), 3.92$ (t, $\left.J=6.1 \mathrm{~Hz}, 2 \mathrm{H}, \mathrm{OCH}_{2}\right), 7.57$ (s, 2H, $\left.2 \times \mathrm{Ar}-\mathrm{H}\right)$.

参照上述操作, 化合物 $27.186 \mathrm{~g}$ (19.98 mmol), 溴 代十四烷 $5.522 \mathrm{~g}$ (20.01 mmol)得无色液体 7b $11.04 \mathrm{~g}$, 收率 99.23\%. ${ }^{1} \mathrm{H} \mathrm{NMR}\left(\mathrm{CDCl}_{3}, 500 \mathrm{~Hz}\right) \delta: 0.88(\mathrm{t}, J=7.0$ $\left.\mathrm{Hz}, 3 \mathrm{H}, \mathrm{CH}_{3}\right), 1.20 \sim 1.42\left(\mathrm{br}, 20 \mathrm{H}, 10 \times \mathrm{CH}_{2}\right), 1.49 \sim 1.58$ $\left(\mathrm{m}, 2 \mathrm{H}, \mathrm{CH}_{2}\right), 1.84 \sim 1.95\left(\mathrm{~m}, 2 \mathrm{H}, \mathrm{CH}_{2}\right), 2.23(\mathrm{~s}, 3 \mathrm{H}$, $\left.\mathrm{Ar}-\mathrm{CH}_{3}\right), 3.92\left(\mathrm{t}, J=6.6 \mathrm{~Hz}, 2 \mathrm{H}, \mathrm{OCH}_{2}\right), 7.57(\mathrm{~s}, 2 \mathrm{H}, 2 \times$ Ar-H).

\section{9 半环苯炔衍生物 8 的合成}

在 $50 \mathrm{~mL}$ 单口烧瓶中加入 $6 \mathrm{a} 0.554 \mathrm{~g}(2.15 \mathrm{mmol})$, 7a $5.174 \mathrm{~g}(8.45 \mathrm{mmol})$, 无水 THF $23 \mathrm{~mL}$, 无水三乙胺 $13 \mathrm{~mL}$, 通氮气驱赶氧气 $5 \mathrm{~min}$, 再加入二三苯基膦二氯 化钯 $0.053 \mathrm{~g}$, 三苯基膦 $0.062 \mathrm{~g}$, 碘化亚铜 $0.024 \mathrm{~g}$, 密闭 搅拌 $14 \mathrm{~h}$, 过滤, 柱层析得 $8 \mathbf{a} 0.538 \mathrm{~g}$, 以 $6 \mathbf{a}$ 计收率 $20.40 \%$. ${ }^{1} \mathrm{H} \mathrm{NMR}\left(\mathrm{CDCl}_{3}, 500 \mathrm{~Hz}\right) \delta: 0.90$ (t, $J=6.8 \mathrm{~Hz}$, $\left.3 \mathrm{H}, \mathrm{CH}_{3}\right), 1.15 \sim 1.47$ (br, $\left.28 \mathrm{H}, 14 \times \mathrm{CH}_{2}\right), 1.48 \sim 1.58(\mathrm{~m}$, $\left.2 \mathrm{H}, \mathrm{CH}_{2}\right), 1.85 \sim 1.94\left(\mathrm{~m}, 2 \mathrm{H}, \mathrm{CH}_{2}\right), 2.29(\mathrm{~s}, 6 \mathrm{H}, 2 \times$ $\left.\mathrm{Ar}-\mathrm{CH}_{3}\right), 2.32\left(\mathrm{~s}, 3 \mathrm{H}, \mathrm{Ar}-\mathrm{CH}_{3}\right), 3.31\left(\mathrm{~s}, 3 \mathrm{H}, \mathrm{OCH}_{3}\right), 3.46$ (t, $\left.J=4.8 \mathrm{~Hz}, 2 \mathrm{H}, \mathrm{CH}_{2}\right), 3.70$ (t, $\left.J=4.5 \mathrm{~Hz}, 2 \mathrm{H}, \mathrm{CH}_{2}\right), 3.93$ (t, $\left.J=5.0 \mathrm{~Hz}, 2 \mathrm{H}, \mathrm{CH}_{2}\right), 4.16\left(\mathrm{t}, J=6.3 \mathrm{~Hz}, 4 \mathrm{H}, 2 \times \mathrm{OCH}_{2}\right.$ ), 4.47 (t, $\left.J=5.0 \mathrm{~Hz}, 2 \mathrm{H}, \mathrm{CH}_{2}\right), 7.28$ (s, 2H, $\left.2 \times \mathrm{Ar}-\mathrm{H}\right), 7.30$ $(\mathrm{s}, 2 \mathrm{H}, 2 \times \mathrm{Ar}-\mathrm{H}), 7.60(\mathrm{~s}, 2 \mathrm{H}, 2 \times \mathrm{Ar}-\mathrm{H}) ;{ }^{13} \mathrm{C} \mathrm{NMR}$ $\left(\mathrm{CDCl}_{3}, 125 \mathrm{~Hz}\right) \delta$ : $14.49\left(2 \mathrm{C}, \mathrm{CH}_{3}\right), 20.40,20.79,23.08$, 26.68, 29.76, 30.11, 30.81, 32.33 (35C, $3 \times \mathrm{Ar}^{-\mathrm{CH}_{3}, 32 \times}$ $\left.\mathrm{CH}_{2}\right), 59.33\left(1 \mathrm{C}, \mathrm{OCH}_{3}\right), 70.99,72.33,73.79,74.71$ (4C, $\left.\mathrm{OCH}_{2}\right), 90.14,90.37,92.44(4 \mathrm{C}, \mathrm{C} \equiv \mathrm{C}), 117.58,117.73$, $133.39,134.46,134.63,135.21,140.56,158.00,159.10$
(18C，三个苯环).

参照上述操作, 6b $0.319 \mathrm{~g}$ (1.06 mmol), 7b $2.072 \mathrm{~g}$ (3.73 mmol) 得 $8 b 0.221 \mathrm{~g}$, 以 $6 \mathbf{b}$ 计收率 $18.00 \%$. ${ }^{1} \mathrm{H}$ NMR $\left(\mathrm{CDCl}_{3}, 500 \mathrm{~Hz}\right) \delta: 0.87\left(\mathrm{t}, J=7.0 \mathrm{~Hz}, 3 \mathrm{H}, \mathrm{CH}_{3}\right), 1.15 \sim$ $1.40\left(\mathrm{br}, 20 \mathrm{H}, 10 \times \mathrm{CH}_{2}\right), 1.47 \sim 1.56\left(\mathrm{~m}, 2 \mathrm{H}, \mathrm{CH}_{2}\right), 1.80 \sim$ $1.90\left(\mathrm{~m}, 2 \mathrm{H}, \mathrm{CH}_{2}\right), 2.27\left(\mathrm{~s}, 6 \mathrm{H}, 2 \times \mathrm{Ar}-\mathrm{CH}_{3}\right), 2.30(\mathrm{~s}, 3 \mathrm{H}$, $\left.\mathrm{Ar}-\mathrm{CH}_{3}\right), 3.34\left(\mathrm{~s}, 3 \mathrm{H}, \mathrm{OCH}_{3}\right), 3.47(\mathrm{t}, J=4.9 \mathrm{~Hz}, 2 \mathrm{H}$, $\mathrm{CH}_{2}$ ), 3.54 (br, $4 \mathrm{H}, 2 \times \mathrm{CH}_{2}$ ), 3.69 (t, $J=4.7 \mathrm{~Hz}, 2 \mathrm{H}, \mathrm{CH}_{2}$ ), $3.89\left(\mathrm{t}, J=5.0 \mathrm{~Hz}, 2 \mathrm{H}, \mathrm{CH}_{2}\right), 4.13(\mathrm{t}, J=5.0 \mathrm{~Hz}, 4 \mathrm{H}, 2 \times$ $\left.\mathrm{OCH}_{2}\right), 4.43\left(\mathrm{t}, J=4.9 \mathrm{~Hz}, 2 \mathrm{H}, \mathrm{CH}_{2}\right), 7.26(\mathrm{~s}, 2 \mathrm{H}, 2 \times$ Ar-H), 7.28 (s, 2H, $2 \times$ Ar-H), 7.58 (s, 2H, $2 \times$ Ar-H); ${ }^{13} \mathrm{C}$ NMR $\left(\mathrm{CDCl}_{3}, 125 \mathrm{~Hz}\right) \delta: 14.50\left(2 \mathrm{C}, \mathrm{CH}_{3}\right), 20.43,20.80$, 23.09, 26.68, 29.77, 30.09, 30.81, 32.33 (27C, $3 \times \mathrm{Ar}_{-} \mathrm{CH}_{3}$, $\left.24 \times \mathrm{CH}_{2}\right), 59.38\left(1 \mathrm{C}, \mathrm{OCH}_{3}\right), 70.88,70.95,71.08,72.29$, 73.74, $74.72\left(6 \mathrm{C}, \mathrm{OCH}_{2}\right), 90.12,90.37,92.45(4 \mathrm{C}, \mathrm{C} \equiv \mathrm{C})$, $117.58,117.72,133.40,134.46,134.63,135.24,140.56$, 157.97, 159.29 (18C, 三个苯环).

参照上述操作, 6b $0.532 \mathrm{~g}$ (1.76 mmol), 4b $3.123 \mathrm{~g}$ $(6.17 \mathrm{mmol})$ 得 $8 \mathbf{c} 0.987 \mathrm{~g}$, 以 $6 \mathbf{b}$ 计收率 $52.99 \%$. ${ }^{1} \mathrm{H}$ NMR $\left(\mathrm{CDCl}_{3}, 500 \mathrm{~Hz}\right) \delta: 2.31\left(\mathrm{br}, 9 \mathrm{H}, 3 \times \mathrm{Ar}-\mathrm{CH}_{3}\right), 3.30(\mathrm{~s}, 6 \mathrm{H}$, $\left.2 \times \mathrm{OCH}_{3}\right), 3.45$ (br, $\left.30 \mathrm{H}, 15 \times \mathrm{CH}_{2}\right), 4.35$ (br, $2 \mathrm{H}, \mathrm{CH}_{2}$ ), 4.45 (br, $\left.4 \mathrm{H}, 2 \times \mathrm{CH}_{2}\right), 7.26 \sim 7.70$ (br, $\left.6 \mathrm{H}, 6 \times \mathrm{Ar}-\mathrm{H}\right)$.

\subsection{0 半环苯炔衍生物 9c 的合成}

在 $50 \mathrm{~mL}$ 单口烧瓶中加入 $8 \mathrm{a} 0.490 \mathrm{~g}(0.40 \mathrm{mmol})$, 三甲基硅乙炔 $0.214 \mathrm{~g}(2.18 \mathrm{mmol})$, 无水 THF $7 \mathrm{~mL}$, 无 水三乙胺 $5 \mathrm{~mL}$, 通氮气驱赶氧气 $15 \mathrm{~min}$, 再加入二三 苯基膦二氯化钯 $0.034 \mathrm{~g}$ ，三苯基膦 $0.055 \mathrm{~g}$, 碘化亚铜 $0.028 \mathrm{~g}$, 密闭搅拌 $14 \mathrm{~h}$, 过滤, 柱层析得 9a $0.477 \mathrm{~g}$, 以 8a 计收率 97.22\%. ${ }^{1} \mathrm{H}$ NMR $\left(\mathrm{CDCl}_{3}, 500 \mathrm{~Hz}\right) \delta: 0.28(\mathrm{~s}$, $\left.18 \mathrm{H}, 6 \times \mathrm{Si}_{-}-\mathrm{CH}_{3}\right), 0.91\left(\mathrm{t}, J=6.9 \mathrm{~Hz}, 3 \mathrm{H}, \mathrm{CH}_{3}\right), 1.10 \sim$ $1.47\left(\mathrm{br}, 56 \mathrm{H}, 28 \times \mathrm{CH}_{2}\right), 1.50 \sim 1.60\left(\mathrm{~m}, 2 \mathrm{H}, \mathrm{CH}_{2}\right), 1.82 \sim$ $1.89\left(\mathrm{~m}, 2 \mathrm{H}, \mathrm{CH}_{2}\right), 2.29\left(\mathrm{~s}, 6 \mathrm{H}, 2 \times \mathrm{Ar}-\mathrm{CH}_{3}\right), 2.32(\mathrm{~s}, 3 \mathrm{H}$, $\left.\mathrm{Ar}-\mathrm{CH}_{3}\right), 3.31\left(\mathrm{~s}, 3 \mathrm{H}, \mathrm{OCH}_{3}\right), 3.47(\mathrm{t}, J=5.0 \mathrm{~Hz}, 2 \mathrm{H}$, $\left.\mathrm{CH}_{2}\right), 3.72\left(\mathrm{t}, J=5.0 \mathrm{~Hz}, 2 \mathrm{H}, \mathrm{CH}_{2}\right), 3.95(\mathrm{t}, J=5.0 \mathrm{~Hz}, 2 \mathrm{H}$, $\left.\mathrm{CH}_{2}\right), 4.25\left(\mathrm{t}, J=6.5 \mathrm{~Hz}, 4 \mathrm{H}, 2 \times \mathrm{OCH}_{2}\right), 4.50(\mathrm{t}, J=5.1$ $\left.\mathrm{Hz}, 2 \mathrm{H}, \mathrm{CH}_{2}\right), 7.25(\mathrm{~s}, 2 \mathrm{H}, 2 \times \mathrm{Ar}-\mathrm{H}), 7.28(\mathrm{~s}, 2 \mathrm{H}, 2 \times$ $\mathrm{Ar}-\mathrm{H}), 7.30$ (s, $2 \mathrm{H}, 2 \times \mathrm{Ar}-\mathrm{H}) ;{ }^{13} \mathrm{C} \mathrm{NMR}\left(\mathrm{CDCl}_{3}, 125 \mathrm{~Hz}\right)$ $\delta$ : $0.33\left(6 \mathrm{C}, \mathrm{SiCH}_{3}\right), 14.49\left(2 \mathrm{C}, \mathrm{CH}_{3}\right), 20.69,23.09,26.67$, 29.76, 30.11, 30.93, 32.33 (35C, $\left.3 \times \mathrm{Ar}-\mathrm{CH}_{3}, 32 \times \mathrm{CH}_{2}\right)$, $59.29\left(1 \mathrm{C}, \mathrm{OCH}_{3}\right), 70.99,72.35,73.73,74.86\left(4 \mathrm{C}, \mathrm{OCH}_{2}\right)$, 89.83, 90.33, 98.95, 101.62 (8C, $\mathrm{C} \equiv \mathrm{C}), 117.69$, 117.90, $132.95,133.00,134.37,134.62,134.90,159.22,160.01$ (18C, 三个苯环).

参照上述操作, 8b 0.221 g (0.19 mmol)得 9b $0.199 \mathrm{~g}$, 以 $8 \mathrm{~b}$ 计收率 $95.30 \% .{ }^{1} \mathrm{H} \mathrm{NMR}\left(\mathrm{CDCl}_{3}, 500 \mathrm{~Hz}\right) \delta: 0.29(\mathrm{~s}$, 
$\left.18 \mathrm{H}, 6 \times \mathrm{Si}^{-\mathrm{CH}_{3}}\right), 0.89\left(\mathrm{t}, J=7.0 \mathrm{~Hz}, 3 \mathrm{H}, \mathrm{CH}_{3}\right), 1.10 \sim$ 1.40 (br, $\left.40 \mathrm{H}, 20 \times \mathrm{CH}_{2}\right), 1.49 \sim 1.59\left(\mathrm{~m}, 2 \mathrm{H}, \mathrm{CH}_{2}\right), 1.80 \sim$ $1.90\left(\mathrm{~m}, 2 \mathrm{H}, \mathrm{CH}_{2}\right), 2.29\left(\mathrm{~s}, 6 \mathrm{H}, 2 \times \mathrm{Ar}-\mathrm{CH}_{3}\right), 2.32(\mathrm{~s}, 3 \mathrm{H}$, Ar- $\left.\mathrm{CH}_{3}\right), 3.36\left(\mathrm{~s}, 3 \mathrm{H}, \mathrm{OCH}_{3}\right), 3.49$ (t, $J=2.9 \mathrm{~Hz}, 2 \mathrm{H}$, $\mathrm{CH}_{2}$ ), 3.57 (br, $\left.4 \mathrm{H}, 2 \times \mathrm{CH}_{2}\right), 3.72\left(\mathrm{t}, J=5.1 \mathrm{~Hz}, 2 \mathrm{H}, \mathrm{CH}_{2}\right)$, $3.93\left(\mathrm{t}, 2 \mathrm{H}, \mathrm{CH}_{2}\right), 4.25$ (t, $\left.J=6.4 \mathrm{~Hz}, 4 \mathrm{H}, 2 \times \mathrm{OCH}_{2}\right), 4.48$ (t, $\left.2 \mathrm{H}, \mathrm{CH}_{2}\right), 7.25$ (s, 2H, $\left.2 \times \mathrm{Ar}-\mathrm{H}\right), 7.28(\mathrm{~s}, 2 \mathrm{H}, 2 \times$ $\mathrm{Ar}-\mathrm{H}), 7.29$ (s, $2 \mathrm{H}, 2 \times \mathrm{Ar}-\mathrm{H}) ;{ }^{13} \mathrm{C} \mathrm{NMR}\left(\mathrm{CDCl}_{3}, 125 \mathrm{~Hz}\right)$ $\delta: 0.33\left(6 \mathrm{C}, \mathrm{SiCH}_{3}\right), 14.50\left(2 \mathrm{C}, \mathrm{CH}_{3}\right), 19.57,23.09,26.68$, $29.78,30.12,30.94,32.34\left(27 \mathrm{C}, 3 \times \mathrm{Ar}^{-} \mathrm{CH}_{3}, 24 \times \mathrm{CH}_{2}\right)$, $59.33\left(1 \mathrm{C}, \mathrm{OCH}_{3}\right), 70.86,70.98,71.08,72.28,73.68,74.86$ $\left(6 \mathrm{C}, \mathrm{OCH}_{2}\right), 89.83,90.30,98.96,101.59(8 \mathrm{C}, \mathrm{C} \equiv \mathrm{C})$, $117.70,117.85,117.89,132.97,133.32,134.37,134.62$, $134.91,159.22,159.99$ (18C, 三个苯环).

参照上述操作, 8c $0.987 \mathrm{~g}(0.93 \mathrm{mmol})$ 得 $9 \mathrm{c} 0.304 \mathrm{~g}$, 以 8c 计收率 $32.72 \% .{ }^{1} \mathrm{H} \mathrm{NMR}\left(\mathrm{CDCl}_{3}, 500 \mathrm{~Hz}\right) \delta: 0.27$ (s, $\left.18 \mathrm{H}, 6 \times \mathrm{SiCH}_{3}\right), 2.28\left(\mathrm{~s}, 6 \mathrm{H}, 2 \times \mathrm{ArCH}_{3}\right), 2.31(\mathrm{~s}, 3 \mathrm{H}$, $\left.\mathrm{ArCH}_{3}\right), 3.34\left(\mathrm{~s}, 3 \mathrm{H}, \mathrm{OCH}_{3}\right), 3.35\left(\mathrm{~s}, 6 \mathrm{H}, 2 \times \mathrm{OCH}_{3}\right)$, $3.48 \sim 3.80$ (br, $24 \mathrm{H}, 12 \times \mathrm{CH}_{2}$ ), $3.91(\mathrm{t}, J=8.5 \mathrm{~Hz}, 6 \mathrm{H}, 3 \times$ $\left.\mathrm{CH}_{2}\right), 4.42\left(\mathrm{t}, J=8.5 \mathrm{~Hz}, 4 \mathrm{H}, 2 \times \mathrm{CH}_{2}\right), 4.49\left(\mathrm{t}, 2 \mathrm{H}, \mathrm{CH}_{2}\right)$, $7.18 \sim 7.30$ (br, $6 \mathrm{H}, 6 \times \mathrm{Ar}-\mathrm{H}$ ).

\subsection{1 苯炔半环 10 的合成}

在 $25 \mathrm{~mL}$ 单口烧瓶中加入 9a $0.477 \mathrm{~g}(0.41 \mathrm{mmol})$, 无水 THF $8 \mathrm{~mL}$, 无水甲醇 $7 \mathrm{~mL}$, 无水碳酸钾 $2.500 \mathrm{~g}$, 搅拌 $3 \mathrm{~h}$, 过滤, 滤液蒸干, 柱层析得 10a $0.400 \mathrm{~g}$, 收率 95.38\%. ${ }^{1} \mathrm{H} \mathrm{NMR}\left(\mathrm{CDCl}_{3}, 500 \mathrm{~Hz}\right) \delta: 0.88(\mathrm{t}, J=7.1 \mathrm{~Hz}$, $\left.3 \mathrm{H}, \mathrm{CH}_{3}\right), 1.10 \sim 1.42\left(\mathrm{br}, 56 \mathrm{H}, 28 \times \mathrm{CH}_{2}\right), 1.43 \sim 1.55(\mathrm{~m}$, $\left.2 \mathrm{H}, \mathrm{CH}_{2}\right), 1.76 \sim 1.86\left(\mathrm{~m}, 2 \mathrm{H}, \mathrm{CH}_{2}\right), 2.28(\mathrm{~s}, 6 \mathrm{H}, 2 \times$ $\mathrm{ArCH}_{3}$ ), 2.30 (s, $\left.3 \mathrm{H}, \mathrm{ArCH}_{3}\right), 3.23$ (s, $\left.2 \mathrm{H}, 2 \times \mathrm{CH}\right), 3.28$ (s, $\left.3 \mathrm{H}, \mathrm{OCH}_{3}\right), 3.44\left(\mathrm{t}, J=4.9 \mathrm{~Hz}, 2 \mathrm{H}, \mathrm{CH}_{2}\right), 3.69$ (t, $J=5.0$ $\left.\mathrm{Hz}, 2 \mathrm{H}, \mathrm{CH}_{2}\right), 3.92$ (t, $\left.J=4.9 \mathrm{~Hz}, 2 \mathrm{H}, \mathrm{CH}_{2}\right), 4.23$ (t, $J=6.5$ $\left.\mathrm{Hz}, 4 \mathrm{H}, 2 \times \mathrm{OCH}_{2}\right), 4.47\left(\mathrm{t}, J=5.0 \mathrm{~Hz}, 2 \mathrm{H}, \mathrm{CH}_{2}\right), 7.24$ (s, $2 \mathrm{H}, 2 \times \mathrm{Ar}-\mathrm{H}), 7.26(\mathrm{~s}, 2 \mathrm{H}, 2 \times \mathrm{Ar}-\mathrm{H}), 7.30$ (s, $2 \mathrm{H}, 2 \times$ $\mathrm{Ar}-\mathrm{H}) ;{ }^{13} \mathrm{C} \mathrm{NMR}\left(\mathrm{CDCl}_{3}, 125 \mathrm{~Hz}\right) \delta: 14.48\left(2 \mathrm{C}, \mathrm{CH}_{3}\right)$, 20.70 (3C, $\left.\mathrm{Ar}-\mathrm{CH}_{3}\right), 23.08,26.56,29.75,30.10,30.82$, $32.32\left(32 \mathrm{C}, \mathrm{CH}_{2}\right), 59.29\left(1 \mathrm{C}, \mathrm{OCH}_{3}\right), 70.99,72.33,73.74$, $75.00\left(4 \mathrm{C}, \mathrm{OCH}_{2}\right), 80.35,81.47,90.05,90.22(8 \mathrm{C}, \mathrm{C} \equiv \mathrm{C})$, $116.79,117.83,117.90,133.09,134.40,135.00,135.14$, $159.00,160.19$ (18C, 三个苯环).

参照上述操作, 9b $0.199 \mathrm{~g}(0.18 \mathrm{mmol})$ 得 $\mathbf{1 0 b} 0.143$ $\mathrm{g}$, 以 $8 \mathrm{c}$ 计收率 $82.83 \% .{ }^{1} \mathrm{H} \mathrm{NMR}\left(\mathrm{CDCl}_{3}, 500 \mathrm{~Hz}\right) \delta: 0.87$ (t, $\left.J=6.9 \mathrm{~Hz}, 3 \mathrm{H}, \mathrm{CH}_{3}\right), 1.10 \sim 1.40\left(\mathrm{br}, 40 \mathrm{H}, 20 \times \mathrm{CH}_{2}\right.$ ), $1.44 \sim 1.53\left(\mathrm{~m}, 2 \mathrm{H}, \mathrm{CH}_{2}\right), 1.76 \sim 1.86\left(\mathrm{~m}, 2 \mathrm{H}, \mathrm{CH}_{2}\right), 2.28$ (s, $\left.6 \mathrm{H}, 2 \times \mathrm{ArCH}_{3}\right), 2.30\left(\mathrm{~s}, 3 \mathrm{H}, \mathrm{ArCH}_{3}\right), 3.24(\mathrm{~s}, 2 \mathrm{H}, 2 \times$ $\mathrm{CH}), 3.33\left(\mathrm{~s}, 3 \mathrm{H}, \mathrm{OCH}_{3}\right), 3.47\left(\mathrm{t}, J=5.0 \mathrm{~Hz}, 2 \mathrm{H}, \mathrm{CH}_{2}\right)$,
3.54 (br, $4 \mathrm{H}, 2 \times \mathrm{CH}_{2}$ ), 3.69 (t, $J=4.5 \mathrm{~Hz}, 2 \mathrm{H}, \mathrm{CH}_{2}$ ), 3.90 (t, $\left.J=4.9 \mathrm{~Hz}, 2 \mathrm{H}, \mathrm{CH}_{2}\right), 4.23(\mathrm{t}, J=6.4 \mathrm{~Hz}, 4 \mathrm{H}, 2 \times$ $\left.\mathrm{OCH}_{2}\right), 4.45\left(\mathrm{t}, J=5.0 \mathrm{~Hz}, 2 \mathrm{H}, \mathrm{CH}_{2}\right), 7.25(\mathrm{~s}, 2 \mathrm{H}, 2 \times$ Ar-H), 7.26 (s, 2H, $2 \times$ Ar-H), 7.30 (s, 2H, $2 \times$ Ar-H); ${ }^{13} \mathrm{C}$ NMR $\left(\mathrm{CDCl}_{3}, 125 \mathrm{~Hz}\right) \delta$ : $14.49\left(2 \mathrm{C}, \mathrm{CH}_{3}\right), 20.73(3 \mathrm{C}$, $\left.\mathrm{Ar}-\mathrm{CH}_{3}\right), 23.08,26.56,29.76,30.10,30.82,32.33$ (24C, $\left.\mathrm{CH}_{2}\right), 59.35\left(1 \mathrm{C}, \mathrm{OCH}_{3}\right), 70.86,70.97,71.08,72.28,73.69$, $75.01\left(6 \mathrm{C}, \mathrm{OCH}_{2}\right), 80.33,81.50,90.05,90.20(8 \mathrm{C}, \mathrm{C} \equiv \mathrm{C})$, $116.79,117.83,117.90,133.11,133.29,134.40,135.00$, $135.14,159.27,160.17$ (18C, 三个苯环).

参照上述操作, 9c $0.304 \mathrm{~g}(0.30 \mathrm{mmol})$ 得 10c 0.251 $\mathrm{g}$, 以 $8 \mathrm{c}$ 计收率 $97.84 \% .{ }^{1} \mathrm{H} \mathrm{NMR}\left(\mathrm{CDCl}_{3}, 500 \mathrm{~Hz}\right) \delta: 2.30$ (s, $\left.6 \mathrm{H}, 2 \times \mathrm{ArCH}_{3}\right), 2.32$ (s, $\left.3 \mathrm{H}, \mathrm{ArCH}_{3}\right), 3.31$ (s, $2 \mathrm{H}, 2 \times$ $\mathrm{CH}), 3.35$ (s, $\left.3 \mathrm{H}, \mathrm{OCH}_{3}\right), 3.37\left(\mathrm{~s}, 6 \mathrm{H}, 2 \times \mathrm{OCH}_{3}\right), 3.48 \sim$ $3.80\left(\mathrm{br}, 24 \mathrm{H}, 12 \times \mathrm{CH}_{2}\right), 3.90 \sim 4.50\left(\mathrm{br}, 6 \mathrm{H}, 3 \times \mathrm{CH}_{2}\right)$, $7.23 \sim 7.35$ (br, $6 \mathrm{H}, 6 \times \mathrm{Ar}-\mathrm{H})$.

\subsection{2 苯炔大环 11 的合成}

10a $0.069 \mathrm{~g}$ 溶于 $90 \mathrm{~mL}$ 无水吡啶, 置于 $100 \mathrm{~mL}$ 恒 压漏斗中. 在 $100 \mathrm{~mL}$ 单口烧瓶中加入 $0.258 \mathrm{~g}$ 无水氯化 亚铜, $0.051 \mathrm{~g}$ 无水氯化铜, $15 \mathrm{~mL}$ 无水吡啶, 密闭搅拌下 十分缓慢地滴入 $10 \mathrm{a}$ 的无水吡啶溶液, $96 \mathrm{~h}$ 滴完, 然后摚 拌 $24 \mathrm{~h}$, 移到 $250 \mathrm{~mL}$ 分液漏斗中, 加入 $40 \mathrm{~mL}$ 三氯甲烷 和 $30 \mathrm{~mL}$ 浓氨水, 摇动, 静置, 下层 $50 \mathrm{~mL}$ 蒸馏水洗一 次, 干燥, 水浴减压蒸去三氯甲烷和残留吡啶, 制备型 薄层分离得棕色固体 11a $0.034 \mathrm{~g}$, 收率 49.28\%. m.p. 125 ${ }^{\circ} \mathrm{C}$; ${ }^{1} \mathrm{H} \mathrm{NMR}\left(\mathrm{CDCl}_{3}, 500 \mathrm{~Hz}\right) \delta: 0.87(\mathrm{t}, J=6.7 \mathrm{~Hz}, 12 \mathrm{H}$, $\left.4 \times \mathrm{CH}_{3}\right), 1.10 \sim 1.40\left(\mathrm{br}, 112 \mathrm{H}, 56 \times \mathrm{CH}_{2}\right), 1.40 \sim 1.50(\mathrm{~m}$, $\left.8 \mathrm{H}, 4 \times \mathrm{CH}_{2}\right), 1.78 \sim 1.86\left(\mathrm{~m}, 8 \mathrm{H}, 4 \times \mathrm{CH}_{2}\right), 2.28(\mathrm{~s}, 12 \mathrm{H}$, $\left.4 \times \mathrm{ArCH}_{3}\right), 2.30\left(\mathrm{~s}, 6 \mathrm{H}, 2 \times \mathrm{ArCH}_{3}\right), 3.28(\mathrm{~s}, 6 \mathrm{H}, 2 \times$ $\mathrm{OCH}_{3}$ ), 3.31 (t, $\left.J=4.1 \mathrm{~Hz}, 4 \mathrm{H}, 2 \times \mathrm{CH}_{2}\right), 3.49$ (t, $4 \mathrm{H}, 2 \times$ $\mathrm{CH}_{2}$ ), 3.86 (t, $\left.4 \mathrm{H}, 2 \times \mathrm{CH}_{2}\right), 4.36(\mathrm{t}, J=6.4 \mathrm{~Hz}, 8 \mathrm{H}, 4 \times$ $\left.\mathrm{OCH}_{2}\right), 4.56\left(\mathrm{t}, 4 \mathrm{H}, 2 \times \mathrm{CH}_{2}\right), 7.23(\mathrm{~s}, 4 \mathrm{H}, 4 \times \mathrm{Ar}-\mathrm{H}), 7.24$ (s, $4 \mathrm{H}, 4 \times \mathrm{Ar}-\mathrm{H}), 7.27$ (s, $4 \mathrm{H}, 4 \times \mathrm{Ar}-\mathrm{H}) ;{ }^{13} \mathrm{C}$ NMR $\left(\mathrm{CDCl}_{3}, 125 \mathrm{~Hz}\right) \delta: 14.47\left(4 \mathrm{C}, \mathrm{CH}_{3}\right), 20.75$ (6C, $\left.\mathrm{Ar}-\mathrm{CH}_{3}\right)$, $23.07,26.51,29.76,30.00,30.08,30.19,30.58,32.32$ $\left(64 \mathrm{C}, \mathrm{CH}_{2}\right), 59.17\left(2 \mathrm{C}, \mathrm{OCH}_{3}\right), 700.71,71.12,72.12,73.76$ $\left(8 \mathrm{C}\right.$, 寡聚醚链上的 $\left.\mathrm{OCH}_{2}\right), 75.26(4 \mathrm{C}$, 与氧原子相连的 碳链上的 $\left.\mathrm{OCH}_{2}\right), 78.52,79.15,90.29,91.01(16 \mathrm{C}, \mathrm{C} \equiv \mathrm{C})$, $116.69,117.74,117.95,132.76,133.77,134.29,134.83$, $160.09,161.94$ (36C, 六个苯环). HRMS calcd for $\mathrm{C}_{140} \mathrm{H}_{200} \mathrm{O}_{10} 2043.5304\left(\mathrm{M}^{+}+1\right)$, found 2043.5304.

参照上述操作, 10b $0.088 \mathrm{~g}$ 得 11b $0.024 \mathrm{~g}$, 收率 27.27\%. m.p. $125{ }^{\circ} \mathrm{C} ;{ }^{1} \mathrm{H}$ NMR $\left(\mathrm{CDCl}_{3}, 500 \mathrm{~Hz}\right) \delta: 0.85$ (t, $\left.J=5.7 \mathrm{~Hz}, 12 \mathrm{H}, 4 \times \mathrm{CH}_{3}\right), 1.00 \sim 1.30\left(\mathrm{br}, 80 \mathrm{H}, 40 \times \mathrm{CH}_{2}\right)$, $1.40 \sim 1.50\left(\mathrm{~m}, 8 \mathrm{H}, 4 \times \mathrm{CH}_{2}\right), 1.78 \sim 1.80\left(\mathrm{~m}, 8 \mathrm{H}, 4 \times \mathrm{CH}_{2}\right)$, 
$2.29\left(\mathrm{~s}, 12 \mathrm{H}, 4 \times \mathrm{ArCH}_{3}\right), 2.30\left(\mathrm{~s}, 6 \mathrm{H}, 2 \times \mathrm{ArCH}_{3}\right), 3.29(\mathrm{~s}$, $\left.6 \mathrm{H}, 2 \times \mathrm{OCH}_{3}\right), 3.33 \sim 3.51\left(\mathrm{br}, 16 \mathrm{H}, 8 \times \mathrm{CH}_{2}\right), 3.84(\mathrm{t}, 4 \mathrm{H}$, $\left.2 \times \mathrm{CH}_{2}\right), 4.37\left(\mathrm{t}, 8 \mathrm{H}, 4 \times \mathrm{OCH}_{2}\right), 4.56(\mathrm{t}, J=\mathrm{Hz}, 4 \mathrm{H}, 2 \times$ $\mathrm{CH}^{2}$ ), 7.24 (s, 8H, $\left.4 \times \mathrm{Ar}-\mathrm{H}\right), 7.28(\mathrm{~s}, 4 \mathrm{H}, 4 \times \mathrm{Ar}-\mathrm{H}) ;{ }^{13} \mathrm{C}$ NMR $\left(\mathrm{CDCl}_{3}, 500 \mathrm{~Hz}\right) \delta$ : $14.55\left(4 \mathrm{C}, \mathrm{CH}_{3}\right), 20.82(6 \mathrm{C}$, $\left.\mathrm{Ar}-\mathrm{CH}_{3}\right), 23.13,26.53,29.85,30.04,30.15,30.26,30.61$, $32.38\left(48 \mathrm{C}, \mathrm{CH}_{2}\right), 59.32\left(2 \mathrm{C}, \mathrm{OCH}_{3}\right), 70.71,70.86,71.32$, $72.23,73.75\left(12 \mathrm{C}\right.$, 寡聚醚链上的 $\left.\mathrm{OCH}_{2}\right), 75.28$ (4C, 与 氧原子相连的碳链上的 $\left.\mathrm{OCH}_{2}\right), 78.49,79.10,90.25$, 91.09 (16C， C $\equiv \mathrm{C}), \quad 116.69 ， 117.68 ， 117.95 ， 132.87$, $133.77,134.33,134.85,161.00,161.87$ (36C, 六个苯环). HRMS calcd for $\mathrm{C}_{128} \mathrm{H}_{176} \mathrm{O}_{12} 1907.3357\left(\mathrm{M}^{+}+1\right)$, found 1907.3357.

参照上述操作，10c $0.066 \mathrm{~g}$ 得 11c $0.024 \mathrm{~g}$, 收率 36.36\%. m.p. $149{ }^{\circ} \mathrm{C} ;{ }^{1} \mathrm{H}$ NMR $\left(\mathrm{CDCl}_{3}, 500 \mathrm{~Hz}\right) \delta: 2.30$ $\left(\mathrm{s}, 12 \mathrm{H}, 4 \times \mathrm{ArCH}_{3}\right), 2.31\left(\mathrm{~s}, 6 \mathrm{H}, 2 \times \mathrm{ArCH}_{3}\right), 3.27(\mathrm{~s}, 12 \mathrm{H}$, $\left.4 \times \mathrm{OCH}_{3}\right), 3.28\left(\mathrm{~s}, 6 \mathrm{H}, 2 \times \mathrm{OCH}_{3}\right), 3.41(\mathrm{t}, J=4.6 \mathrm{~Hz}$, $\left.12 \mathrm{H}, 6 \times \mathrm{CH}_{2}\right), 3.48\left(\mathrm{t}, J=3.9 \mathrm{~Hz}, 12 \mathrm{H}, 6 \times \mathrm{CH}_{2}\right), 3.52(\mathrm{t}$, $\left.12 \mathrm{H}, 6 \times \mathrm{CH}_{2}\right), 3.61\left(\mathrm{t}, J=4.5 \mathrm{~Hz}, 12 \mathrm{H}, 6 \times \mathrm{CH}_{2}\right), 3.87(\mathrm{t}$, $\left.J=4.2 \mathrm{~Hz}, 12 \mathrm{H}, 6 \times \mathrm{CH}_{2}\right), 4.50 \sim 4.63\left(\mathrm{br}, 12 \mathrm{H}, 6 \times \mathrm{CH}_{2}\right)$, $7.17 \sim 7.30$ (br, $12 \mathrm{H}, 12 \times \mathrm{Ar}-\mathrm{H}) ;{ }^{13} \mathrm{C} \mathrm{NMR}\left(\mathrm{CDCl}_{3}, 125\right.$ $\mathrm{Hz}) \delta: 20.75\left(6 \mathrm{C}, \mathrm{ArCH}_{3}\right), 59.20\left(2 \mathrm{C}, \mathrm{OCH}_{3}\right), 64.11(4 \mathrm{C}$, $\left.\mathrm{OCH}_{3}\right), 70.72 \sim 73.94\left(36 \mathrm{C}, \mathrm{OCH}_{2}\right), 78.45,79.13,90.28$, $91.15(16 \mathrm{C}, \mathrm{C} \equiv \mathrm{C}), \quad 116.54,117.72,117.76,132.91$, $133.09,133.82,134.31,134.76,160.72,161.90(36 \mathrm{C}$, 六 个苯环). HRMS calcd for $\mathrm{C}_{100} \mathrm{H}_{120} \mathrm{O}_{24} 1706.8326\left(\mathrm{M}^{+}+\right.$ 1), found 17006.8326 .

\section{2 结果与讨论}

\section{1 关于目标大环的合成、结构及纯度}

ArI 与三甲基硅乙炔反应中，总是存在炔自偶副联 产物, 如果目标产物极性与它相近就可能无法完全分 离，造成反应收率大于 $100 \%$ 的情况出现，当脱去保护 后，因丁二炔沸点太低而自然除去; 化合物 $\mathbf{3 a}, \mathbf{3 b}, \mathbf{4 a}$, $4 b, 8 a, 8 b, 8 c$ 均或多或少地存在室温、空气中不稳定的 情况, 因此, 分离得到目标物后应尽量立即进行下一步 反应，如果需要放置一段时间，则应在氮气气氛下冷藏 保存; 半环闭环合成大环反应中, 为避免聚合反应发生, 反应需在高度稀释下缓慢滴加进行; 该反应对溶剂无水 条件要求较高, 反应溶剂吡啶需用氢化钲回流后重蒸才 能使用; 由于寡聚乙氧基醚链的引入，分子极性增加， 能吸附正离子，因此用 ESI 离子源成功获得高分辩质谱.

本文测定了目标苯炔大环的 UV 和 PL (UV 最强吸 收 $\lambda_{\text {max }}=280 \mathrm{~nm}$ 作为激发光, 发射荧光最强处 $\lambda_{\text {max }}=384$ $\mathrm{nm}$ )(如图 1 所示), 它们的高度相似性表明: 它们具有相
同的大环骨架(内取向的不同基团对苯炔大环骨架共轭 体系几乎不产生影响).
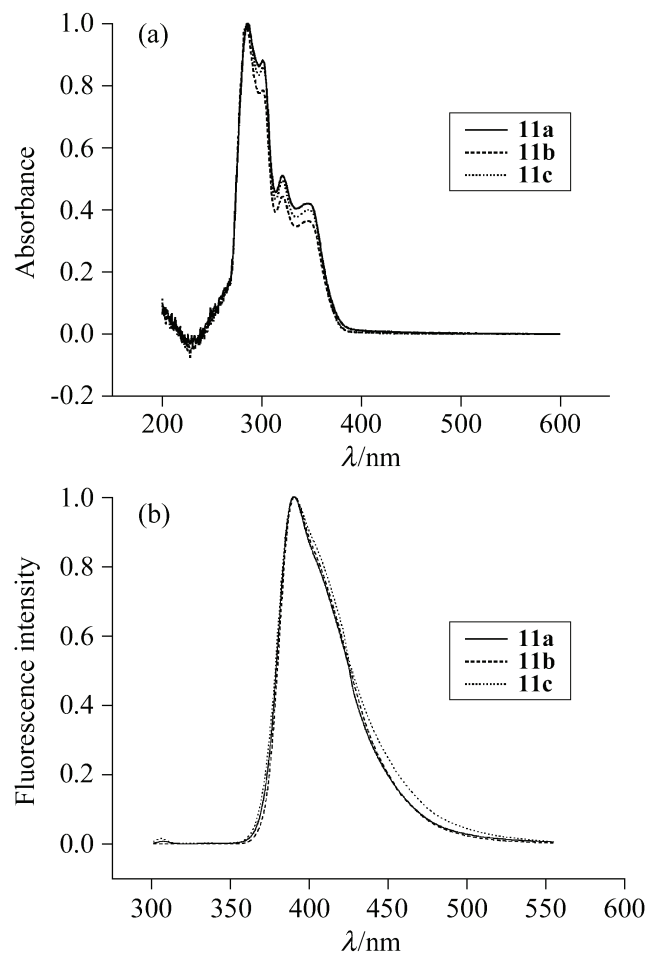

图 $111 \mathrm{a}, 11 \mathrm{~b}$ 和 11c 的 UV 吸收图(a)和荧光发射图(b)

Figure 1 UV (a) and PL (b) curves of 11a, 11b and 11c

本文用 ${ }^{1} \mathrm{H}$ NMR, ${ }^{13} \mathrm{C}$ NMR, UV, PL 和 HRMS 确证了 目标大环的结构, 但是在半环闭环时, 可能产生半环聚 合物, 前述数据难以判断它们是否存在, 本文测定的目 标苯炔大环的凝胶色谱(GPC)清楚表明(如图 2 所示): $11 \mathrm{a}$ 和 $11 \mathrm{~b}$ 为单峰, 无半环聚合物, 11c 因极性太大, 有 少许杂质未能完全分开，但杂质保留时间较大，应为比 $11 \mathrm{c}$ 分子更小的分子, 因此同样可以确定无半环聚合物.

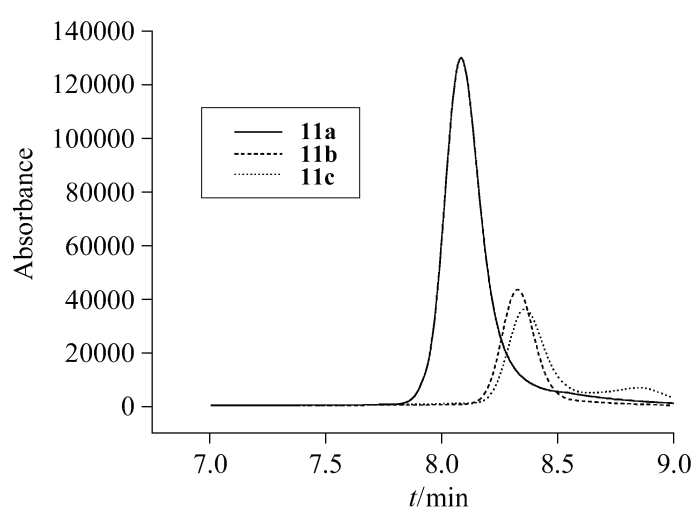

图 $211 \mathrm{a}, 11 \mathrm{~b}$ 和 $11 \mathrm{c}$ 凝胶色谱图

Figure 2 GPC curves of 11a, 11b and 11c

\section{2 非簇集倾向性及不能形成液晶的原因分析}

苯炔大环分子簇集作用力主要来自于 $\pi-\pi$ 作用、溶 
剂化作用、极性基团相互作用，一般形成超分子遂道结 构、二维网状结构或三维结构 ${ }^{[1]}$, 这些结构的基本特征 是苯炔大环骨架同向性的排列. 传统苯炔大环分子的柔 性链处于环骨架外侧, 不会影响苯炔骨架的平面构型, 是推动分子簇集的动力, 但是当苯炔大环分子柔性链处 分子内穴之中, 这种反拓扑结构分子的单晶 $\mathrm{X}$ 衍射表 明：内取向链以一定的倾角处于苯炔大环平面的上下 方 $^{[17]}$, 苯炔大环分子簇集时需要的平面结构不再存在, 内取向链因此成为了环骨架同向性排列的阻力, 如果簇 集作用力不足以促使内取向链平伏于苯炔大环平面内, 分子就无法簇集成具有同向性的有序结构, 因此, 这类 反拓扑结构的分子具有非簇集的倾向性. 即使如本文中 引入了极性寡聚乙氧基醚链来增大分子间作用力, 这种 作用力因其无序性并不会促使内取向链向苯炔平面倾 伏, 对分子簇集成有序结构毫无帮助.

如果分子簇集作用力足够大, 内取向链足够少, 内 取向链可以平伏于苯炔大环平面内, 分子形成同向性有 序结构也就成为可能. Höger 等发现这种反拓扑结构的 分子有的可以形成向列相液晶, 也就是因为熔化时苯炔 分子之间 $\pi-\pi$ 作用可以克服来自于内取向链的阻力, 形 成了仅具方向性的有序结构的原因. Höger 等提出的反 拓扑液晶设计的预备性原则也注意到了能否形成液晶 与内取向的柔性链的多少有关, 但是, Höger等更关注于 对大环内穴的充满情况, 因为内腔未被填满时, 可能出 现一个大环的柔性链穿过另一大环内腔而形成联锁, 从 而不能形成液晶 ${ }^{[18]}$. 可是在环内穴已经足以被内取向链 充满的情况下, 更多的内取向链立于大环平面上下方, 过于拥挤, 无法完全平伏于环平面内形成平面或类似平 面结构. 本文根据 Höger 的预备性原则合成的具有六条 内取向柔性链的苯炔大环 11a, 11b, 11c 就是因为内取向 链太多, 不能形成与苯炔大环共面结构, 所以不能形成 液晶态.

\section{References}

[1] Cheng, X. H.; Ju, X. P.; Höger, S. Chin. J. Org. Chem. 2006, 26(5), 733 (in Chinese).

(程晓红, 鞠秀萍, Sigurd Höger, 有机化学, 2006, 26(5), 733.)

[2] Rosselli, S.; Ramminger, A. D.; Wagner, T.; Silier, B.; Wiegand, S.; Häußler, W.; Lieser, G.; Scheumann, V.; Höger, S. Angew. Chem. 2001, 113, 3233.

[3] Rosselli, S.; Ramminger, A. D.; Wagner, T.; Lieser, G.; Höger, S. Chem. Eur. J. 2003, 9, 3481.

[4] Höger, S.; Morrison, D. L.; Enkelmann, V. J. Am. Chem. Soc. 2002, 124(23), 6734.

[5] Zhang, J. S.; Moore, J. S. J. Am. Chem. Soc. 1994, 116, 2655.

[6] Fischer, M.; Lieser, G.; Rapp, A.; Schnell, I.; Mamdouh, W.; Feyter, S. D.; Schryver, F. C. D.; Höger, S. J. Am. Chem. Soc. 2004, 126, 214.

[7] Kawase, T.; Seirai, Y.; Darabi, H. R.; Oda, M.; Sarakai, Y.; Tashiro, K. Angew Chem. 2003, 115(14), 1662.

[8] Kawase, T.; Nishiyama, Y.; Nakamura, T.; Ebi, T.; Matsumoto, K.; Kurata, H.; Oda. M. Angew Chem. 2007, 119(7), 1104.

[9] Xie, Z.; Zhang, W.; Huang, P. C. Chin. J. Org. Chem. 2002, 22(8), 543 (in Chinese).

(谢政, 张炜, 黄鹏程, 有机化学, 2002, 22(8), 543.)

[10] Moore, J. S. Acc. Chem. Res. 1997, 30, 402.

[11] Höger, S. J. Polym. Sci.: Part A: Polym. Chem. 1999, 37, 2685.

[12] Höger, S. Chem. Eur. J. 2004, 10, 1320.

[13] Cheng, X. H.; Höger, S. Chem. J. Chin. Univ. 2007, 28(1), 65 (in Chinese).

(程晓红, Sigurd Höger, 高等学校化学学报, 2007, 28(1), 65.)

[14] Liu, W. J.; Zhou, Y.; Zhou, Q. F.; Ma, Y. G.; Pei, J. Org. Lett. 2008, 10(11), 2123.

[15] Staab, H. A.; Neunhoeffer, K. Synthesis 1974, 424.

[16] Höger, S.; Enkelmann, V.; Bonrad, K.; Tschiers, C. Angew. Chem., Int. Ed. 2000, 39, 2267.

[17] Höger, S.; Cheng, X. H.; Ramminger, A. D.; Enkelmann, V.; Rapp, A.; Mondeshki; M.; Schnell, I. Angew. Chem., Int. Ed. 2005, 44, 2801.

[18] Höger, S.; Weber, J.; Leppert, A.; Enkelmann, V. Beilstein J. Org. Chem. 2008, $4,1$.

[19] Kouwer, P. H. J.; Jager, W. F.; Mijs, W. J.; Picken, S. J. Macromolecules 2002, 35, 4322.

(Cheng, F.; Zheng, G.) 